\title{
Group-velocity matched nonlinear photonic crystal fibers
}

Bache, Morten; Lægsgaard, Jesper; Bang, Ole

\section{Published in:}

Proceedings ECOC

Link to article, DOI:

10.1109/ECOC.2006.4801110

Publication date:

2006

Document Version

Publisher's PDF, also known as Version of record

Link back to DTU Orbit

Citation (APA):

Bache, M., Lægsgaard, J., \& Bang, O. (2006). Group-velocity matched nonlinear photonic crystal fibers. In Proceedings ECOC (Vol. 2, pp. 139-140). IEEE. https://doi.org/10.1109/ECOC.2006.4801110

\section{General rights}

Copyright and moral rights for the publications made accessible in the public portal are retained by the authors and/or other copyright owners and it is a condition of accessing publications that users recognise and abide by the legal requirements associated with these rights.

- Users may download and print one copy of any publication from the public portal for the purpose of private study or research.

- You may not further distribute the material or use it for any profit-making activity or commercial gain

- You may freely distribute the URL identifying the publication in the public portal

If you believe that this document breaches copyright please contact us providing details, and we will remove access to the work immediately and investigate your claim 


\title{
Group-Velocity Matched Nonlinear Photonic Crystal Fibers
}

Morten Bache, Jesper Lægsgaard, and Ole Bang

COM•DTU, Technical University of Denmark, Bld. 345v, DK-2800 Lyngby, Denmark, bache@com.dtu.dk

\begin{abstract}
A$ quadratic nonlinear index-guiding silica PCF is optimized for efficient second-harmonic generation through dispersion calculations. Zero group-velocity mismatch is possible for any pump wavelength above 780 $\mathrm{nm}$. Very high conversion efficiencies and bandwidths are found.
\end{abstract}

\section{Introduction}

Second-harmonic generation (SHG) is widely used for efficient wavelength conversion devices for extending the spectral range of laser sources and all-optical wavelength multiplexing. Efficient conversion from the fundamental to the second-harmonic $(\mathrm{SH})$ only occurs close to phase matching. To lowest order it is typically achieved through a quasi-phase matching (QPM) technique, so the group-velocity mismatch (GVM) limits the device length and bandwidth for pulsed SHG. For fiber SHG, zero GVM for restricted wavelengths was predicted by changing the core radius [1], and by using mode-matching [2]. In bulk media zero GVM was found for restricted wavelengths by spectrally noncritical phase matching [3] and by combining non-collinear QPM with a pulse-front tilt [4]. Here [5] we investigate efficient pulsed SHG in a silica index-guiding photonic crystal fiber (PCF) with a triangular air-hole pattern and a single-rod core defect (Fig. 1). The PCF design parameters are the pitch $\Lambda$ and the relative hole size $D=d / \Lambda$. We assume a quadratic nonlinearity from thermal poling of the PCF [6]. We tune the phase-matching properties of SHG by exploiting the flexibility that PCFs offer in designing the dispersion properties [7] and maximizing the nonlinear strength. Previous investigations [8] of SHG in PCFs considered the scalar case and found large bandwidths and strong modal overlaps for selected parameter values. Instead, we perform a detailed vectorial analysis over a continuous parameter space, and show zero GVM for any fundamental wavelength $\lambda_{1}>780 \mathrm{~nm}$ by merely adjusting $\Lambda$ and $\mathrm{D}$. This method is much simpler than previous methods [2-4], it shows very large bandwidths, and high efficiency.

\section{Dispersion calculations}

We describe a fiber mode by an effective index $n=c / v_{p h}$, i.e., the ratio of the speed of light $c$ to the phase velocity of the mode $v_{p h}=\omega / \beta$, ( $\beta$ is the propagation constant of the mode.) The dispersive character of $\beta$ gives a phase-velocity mismatch between the fundamental $\left(\omega_{1}\right)$ and $\mathrm{SH}\left(\omega_{2}=2 \omega_{1}\right)$ modes, which we classify through the index mismatch $\Delta \mathrm{n}=\mathrm{n}_{1}$ $\mathrm{n}_{2}=\mathrm{c}\left[1 / \mathrm{v}_{\mathrm{ph}}\left(\omega_{1}\right)-1 / \mathrm{v}_{\mathrm{ph}}\left(\omega_{2}\right)\right]=\mathrm{c}\left(\beta_{1} / \omega_{1}-\beta_{2} / \omega_{2}\right)$, related to the phase mismatch $\Delta \beta=2 \beta_{1}-\beta_{2}$ as $\Delta n=\Delta \beta \lambda_{1} / 4 \pi$. The group velocity is instead defined as $1 / \mathrm{v}_{\mathrm{g}}=\partial \beta / \partial \omega$, giving a GVM (walk-off) parameter $d_{12}=1 / v_{g}\left(\omega_{1}\right)-1 / v_{g}\left(\omega_{2}\right)$. We calculated the fiber modes with the MIT PhotonicBands (MPB) package [9]. First $\omega_{1}$ and $v_{g}\left(\omega_{1}\right)$ were calculated, followed by iterations of the SH until $\mid \omega_{2-}$ $2 \omega_{1} \mid<10^{-8}$. Material dispersion, parameterized by the silica Sellmeier equation, was then included using a perturbative technique [10], whose advantage is that many different $\Lambda$ values can be calculated perturbatively from the MPB data (where $\Lambda$ is unity.)

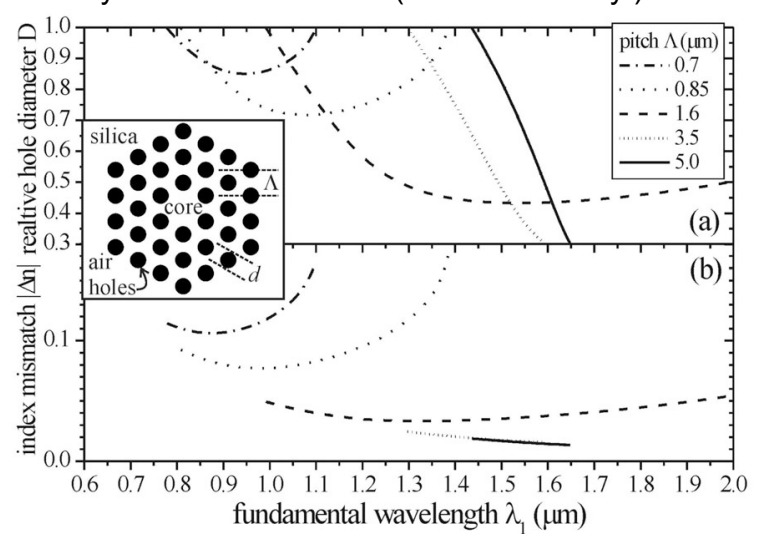

Fig. 1: (a) Zero GVM contours in $\left(\lambda_{1}, D\right)$-space for $\Lambda$ fixed, and (b) $|\Delta n|$ along these contours. Inset: PCF with pitch $\Lambda$ and air-hole diameter $d$.

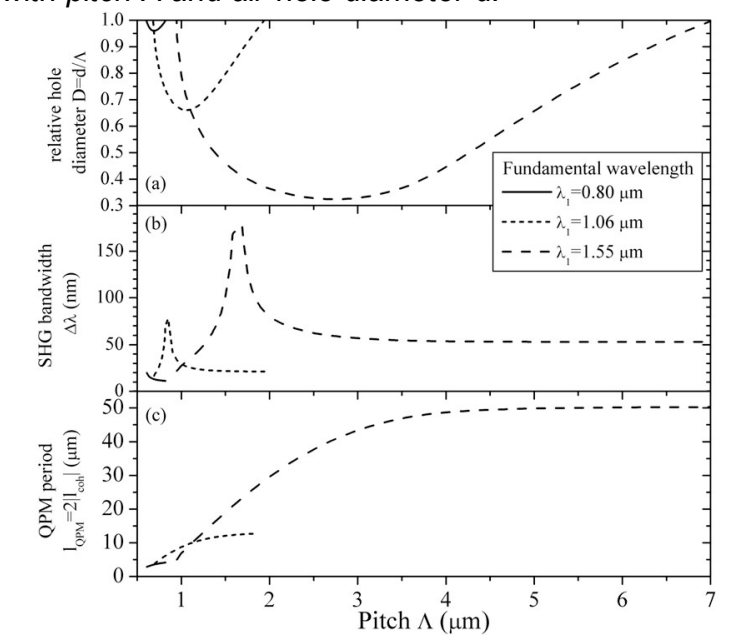

Fig. 2: (a) Zero GVM contours in $(\Lambda, D)$-space and fixing $\lambda_{1}$. (b) and (c) $\Delta \Lambda$ and $l_{Q P M}$ along these contours.

The calculated GVM parameter $d_{12}$ is shown in Fig. 1 (a) as a zero GVM contour $\left(d_{12}=0\right)$ for $0.6 \leq \lambda_{1} \leq 2.0 \mu \mathrm{m}$ and $0.3 \leq D \leq 1$, while keeping the pitch $\wedge$ fixed. The examples show that the design parameters can be tuned over a continuous range to achieve zero GVM for any $\lambda_{1}>0.78 \mu \mathrm{m}$. In Fig.1(b) we show that the corresponding index mismatch $\Delta \mathrm{n}$ is never zero. This is a general trend, even with non-zero GVM, so a QPM method is needed to achieve lowest order phase-matching. In Fig. 1(b) we also note that for 
$\Lambda=0.70,0.85$ and $1.6 \mu \mathrm{m}$ a minimum in $\Delta \mathrm{n}$ appears around $\lambda_{1} \approx \Lambda$. We checked that the core diameter $\mathrm{d}_{\mathrm{c}} \approx \Lambda(2-D)$ relative to $\lambda_{1}$ is maximum there. Thus, when $\lambda_{1}$ increases beyond this point, $d_{d} / \lambda_{1}$ shrinks so the fundamental is less confined in the core. Since the SH stays well-confined (because $\lambda_{2}=\lambda_{1} / 2$ ), an increase in $|\Delta n|$ follows. Focusing on the telecom, $\mathrm{Nd}: Y A G$, and Ti:Sapphire operating wavelengths $\left(\lambda_{1}=1.55,1.06\right.$, and $0.8 \mu \mathrm{m}$, respectively) Fig. 2(a) shows the D-value required to get zero GVM as $\Lambda$ is changed. For $\lambda_{1}=0.80 \mu \mathrm{m}$ zero GVM requires very large $D$-values, e.g., $D=0.96$ for $\Lambda=0.70 \mu \mathrm{m}$. For such $D$-values deviations from the ideal circular holes must be expected, which might influence the results. Instead, for both $\lambda_{1}=1.06$ and $1.55 \mu \mathrm{m}$ the lowest required $D$-values are in a range where the ideal round holes should be preserved. In Fig. 2(b) we calculate the SHG bandwidth $\Delta \lambda$ by expanding $\Delta \beta$ around $\lambda_{1}$ up to third order and assuming that a QPM grating compensates the lowest order term (as in conventional fibers [1]). Since we have $d_{12}=0$, the 2 . order dispersion dominates yielding very large bandwidths. Moreover, because $d_{12}=0, \Delta \lambda$ of a fiber with length $I_{F}$ scales as $I_{F}^{-1 / 2}$ (instead of scaling as $I_{F}{ }^{-1}$ when $\left|d_{12}\right|>0$ ), so a longer device can be created without loosing too much bandwidth. The large peaks in $\Delta \lambda$ can be explained from Fig. 1(a), where a minimum in the zero GVM contour implies that the 2. order term vanishes, giving a very large $\Delta \lambda$ (large enough for fs pulse conversion). Finally, Fig. 2(c) shows the QPM period $\mathrm{I}_{\mathrm{QPM}}=\lambda_{1} /(2|\Delta \mathrm{n}|)$ required to achieve lowest order phase matching. Such values can be reached using periodic poling.

\section{Effective nonlinearity}

Using the reductive perturbation method [11], and assuming that the dimensionless (DL) propagating fields $u_{j}(z, t)$ can be decoupled from the DL transverse MPB modes $\mathbf{e}(\mathbf{x})$, the DL SHG equations are

$$
\begin{aligned}
& \left(\partial_{z}-i D_{1} \partial_{t}^{2}\right) u_{1}=i \sigma u_{1}^{*} u_{2} e^{-i \Delta \beta z l_{F}} \\
& \left(\partial_{z}-d_{12} l_{F} \tau^{-1} \partial_{t}-i D_{2} \partial_{t}^{2}\right) u_{2}=i \sigma u_{1}^{2} e^{i \Delta \beta z l_{F} / 2} \\
& D_{j}=l_{F} /\left(2 \tau^{2}\right) \partial_{\omega}^{2} \beta_{j} \\
& \sigma=\rho l_{F} \sqrt{2 \hbar \omega_{1}^{2} \omega_{2} / n_{1}^{2} n_{2} \varepsilon_{0} c^{3} \tau}, \\
& \rho=\left|\int d \mathbf{x} \mathbf{e}_{1}^{*}(\mathbf{x}) \cdot \tilde{\chi}^{(2)}: \mathbf{e}_{2}(\mathbf{x}) \mathbf{e}_{1}^{*}(\mathbf{x})\right| /\left(\Lambda a_{1} a_{2}^{1 / 2}\right), \\
& a_{j}=\int d \mathbf{x}\left|\mathbf{e}_{j}(\mathbf{x})\right|^{2}
\end{aligned}
$$

$Z$ is scaled to $I_{F}, t$ to the input pulse length $T$, and $\mathbf{x}=(x, y)$ to $\wedge$. Integrating $\left|u_{j}(z, t)\right|^{2}$ over time gives the mode photon number. Fig. 3 shows the nonlinear strength $\sigma$ for $\lambda_{1}$ and $D$ fixed (note that these curves are not zero-GVM contours.) A $2 / \pi$ reduction of $X^{(2)}$ is included because we assume lowest order phase matching through a QPM grating. We found that $\sigma$ scales as $D / \Lambda$, which is due to a smaller core when $\Lambda$ is reduced and a better core confinement when $D$ is increased. $\sigma$ peaks when $\Lambda$ takes values around $\lambda_{1}$, and drops for $\Lambda<\lambda_{1}$ because the fundamental mode has maximum core confinement at the peak [Fig. $3(2)]$. It becomes more poorly confined when $\Lambda<\lambda_{1}$, while the $\mathrm{SH}$ stays better confined [cf. Fig. 3(1)], resulting in a poor modal overlap (controlled by the $\rho$ parameter). The SHG efficiency is $\eta=P_{2}$,out $/ P_{1, \text { in }}$, where $P_{j}$ is the mode power. Depending on the chosen $\lambda_{1}$, we found very large relative efficiencies of 5-180 $\% /\left(\mathrm{W} \mathrm{cm}^{2}\right)$, assuming a realistic $\mathrm{X}^{(2)}=1 \mathrm{pm} / \mathrm{V}$.

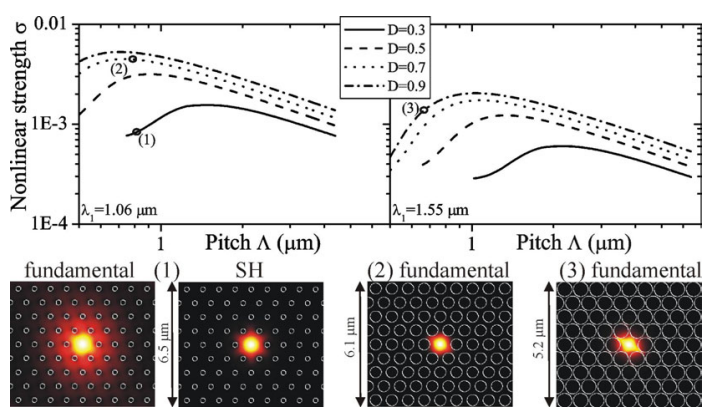

Fig. 3: Double-log plots of $\sigma$ vs. $\Lambda$. (1)-(3): selected mode energy distributions. $l_{F}=10 \mathrm{~cm}, \tau=1 \mathrm{ps}$.

\section{Conclusions}

Tuning the pitch and relative hole size of an indexguiding silica PCF, SHG with zero GVM could be achieved for any $\lambda_{1}>780 \mathrm{~nm}$. The method holds great promise due to its simplicity, and fs pulse-conversion should be feasible. The SHG nonlinear strength was optimized when the fundamental was maximally confined, and it was inversely proportional to the pitch and proportional to the relative hole size. We found up to $180 \% /\left(\mathrm{W} \mathrm{cm}^{2}\right)$ relative conversion efficiencies.

\section{References}

1 P. G. Kazansky et al. J. Opt. Soc. Am. B, 14 (1997), page 3170.

2 A. Arraf et al. IEEE J. Quantum Electron., 34 (1998), page 660.

3 N. E. Yu et al. Opt. Lett., 47 (2002), page 1046.

4 S. Ashihara et al. J. Opt. Soc. Am. B, 20 (2003), page 853.

5 M. Bache et al. arxiv:physics/0511244, to appear in Opt. Lett. (2006).

6 D. Faccio et al. Electron. Lett., 37 (2001), page 107.

7 A. Ferrando et al. Opt. Express, 9 (2000), page 687.

8 T. M. Monro et al. IEEE Photon. Tech. Lett., 13 (2001), page 981.

9 S. Johnson et al. Opt. Express, 8 (2001), page 173.

10J. Lægsgaard et al. J. Opt. Soc. Am. B, 20 (2003), page 443.

11Y. Kodama et al. IEEE J. Quant. Electr., 23 (1987), page 51. 